\title{
DISFAGIA OROFARÍNGEA EM CRIANÇAS COM SÍNDROME CORNÉLIA DE LANGE
}

\section{Oropharyngeal dysphagia in children with Cornelia de Lange syndrome}

\author{
Priscila Martins Foroni (1), Anne Marques Beato ${ }^{(2)}$, \\ Liciane Pinelli Valarelli (3), Luciana Vitaliano Voi Trawitzki ${ }^{(4)}$
}

\begin{abstract}
RESUMO
Objetivo: descrever os achados das fases oral e faríngea da deglutição, bem como os aspectos sensório-motores orofaciais relevantes em crianças com a síndrome Cornélia de Lange. Métodos: tratase de relato de caso, retrospectivo. Realizou-se análise descritiva de quatro protocolos de avaliação fonoaudiológica da deglutição da rotina do Serviço de Fonoaudiologia de crianças com a síndrome Cornélia de Lange, de ambos os sexos, com faixa etária entre 1:2 e 9:6 anos, encaminhadas para avaliação clínica e videofluoroscópica da deglutição em um hospital público universitário. Analisou-se os aspectos das fases oral e faríngea da deglutição por meio da avaliação clínica do sistema sensório-motor orofacial, avaliação clínica funcional e videofluoroscópica. Resultados: As alterações do sistema sensório-motor orofacial mais encontradas foram a hipersensibilidade e a hipertonia muscular da região orofacial. Na fase oral da deglutição, dentre as alterações observadas, destacaram-se a presença de vedamento labial ineficiente, escape oral anterior do bolo alimentar, inadequada formação/organização do bolo e ejeção oral deficiente. Na fase faríngea, observou-se ausculta cervical alterada, refluxo nasal, excursão hiolaríngea reduzida, presença de resíduo em trânsito faríngeo após a deglutição, penetração laríngea e aspiração laringotraqueal. A disfagia orofaríngea foi encontrada em todas as crianças. Conclusão: Os achados encontrados apontaram alterações nos aspectos sensório-motores orofaciais e comprometimento na biomecânica da deglutição. As importantes alterações na deglutição evidenciadas nos casos descritos podem corresponder a manifestações típicas da síndrome Cornélia de Lange.
\end{abstract}

DESCRITORES: Síndrome de Lange; Criança; Deglutição; Transtornos de Deglutição

(1) Fonoaudióloga; Aluna do Curso de Aperfeiçoamento Profissional em Motricidade Orofacial com ênfase em Disfagia Infantil do Hospital das Clínicas da Faculdade de Medicina de Ribeirão Preto da Universidade de São Paulo, HCFMRP-USP, Ribeirão Preto, SP, Brasil.

(2) Fonoaudióloga Assistente do Hospital das Clínicas da Faculdade de Medicina de Ribeirão Preto da Universidade de São Paulo, HCFMRP-USP, Ribeirão Preto, SP, Brasil; Especialista em Audiologia pelo Conselho Federal de Fonoaudiologia.

(3) Fonoaudióloga Assistente do Hospital das Clínicas da Faculdade de Medicina de Ribeirão Preto da Universidade de São Paulo, HCFMRP-USP, Ribeirão Preto, SP, Brasil; Especialista em Motricidade Orofacial pelo Conselho Federal de Fonoaudiologia.

(4) Fonoaudióloga; Docente da Faculdade de Medicina de Ribeirão Preto da Universidade de São Paulo, FMRP. USP, Ribeirão Preto, SP, Brasil; Doutora em Biociências Aplicada à Clínica Médica pela Faculdade de Medicina de Ribeirão Preto da Universidade de São Paulo.

Conflito de interesses: inexistente

\section{INTRODUÇÃO}

A síndrome Cornélia de Lange foi descrita por Brachmann em 1916 e De Lange em 1933 que observaram uma desordem congênita manifestada por anomalias morfológicas da face, deficiência de crescimento pré e pós-natal, atraso do desenvolvimento mental e psicomotor, problemas comportamentais e anomalias de redução dos membros ${ }^{1,2}$. As características faciais na síndrome incluem face inexpressiva com sobrancelhas arqueadas e unidas, nariz pequeno, lábios finos e ligeiramente invertidos, dentição tardia e dentes pequenos, micrognatia, palato arqueado, fissura palatina, orelhas de implantação baixa ${ }^{1-5}$.

Trata-se de uma patologia rara, as estimativas atuais estão entre 1:10.000 e 1:30.000 ${ }^{1,3}$, além de expectativa de vida geralmente baixa, tendo como 
causas de morte mais comuns as apnéias, aspiração de alimentos, anomalias cardíacas, hemorragias intracranianas e incidentes no período pósoperatório ${ }^{6}$.

Em consequência das alterações anatômicas, as funções estomatognáticas de respiração, sucção, mastigação, deglutição e fala também se encontram afetadas ${ }^{7}$.

No período neonatal, o neonato pode apresentar dificuldades de sucção e deglutição, regurgitação, aspiração e episódios de apnéia e cianose ${ }^{3,8}$. Crianças de maior idade tendem a manter a dificuldade de deglutição, como descrito na literatura ${ }^{7,9,10}$. Muitas vezes, a alimentação pode ser prejudicada devido à intensa hipertonia muscular ${ }^{1}$.

O fonoaudiólogo é o profissional capacitado para habilitar e criar alternativas para uma deglutição segura e eficiente. No que diz respeito à síndrome Cornélia de Lange, pouco estudada pela fonoaudiologia, nota-se uma escassez de estudos na literatura que enfoquem a biomecânica da deglutição, o diagnóstico e a atuação fonoaudiológica no que se refere às dificuldades alimentares.

Sendo assim, faz-se necessária a realização de estudos que colaborem para o conhecimento do profissional fonoaudiólogo, visando a qualidade de vida do paciente com essa síndrome e o aperfeiçoamento do atendimento da equipe multidisciplinar.
Desse modo, o objetivo do estudo foi descrever os achados das fases oral e faríngea da deglutição, bem como os aspectos sensório-motores orofaciais relevantes em crianças com a síndrome Cornélia de Lange.

\section{APRESENTAÇÃO DOS CASOS CLÍNICOS}

Trata-se de relato de quatro casos da síndrome Cornélia de Lange, retrospectivo descritivo. Em consulta aos prontuários, realizou-se a análise de protocolos de avaliação fonoaudiológica da deglutição da rotina do Serviço de Fonoaudiologia e das informações da equipe médica de crianças com a síndrome, previamente diagnosticadas, sendo três do gênero feminino e uma do masculino, com faixa etária entre 1:2 e 9:6 anos (média de idade 4,2 anos, mediana de 3 anos).

O estudo foi realizado no Hospital das Clínicas da Faculdade de Medicina de Ribeirão Preto da Universidade de São Paulo - HCFMRP-USP e os pacientes foram encaminhados de diversas clínicas médicas para avaliação fonoaudiológica da deglutição, devido a queixas alimentares.

O Quadro 1 apresenta as características das crianças, com dados de idade, gênero, via de alimentação e histórico de pneumonias, quando encaminhadas para a avaliação fonoaudiológica.

\section{Quadro 1 - Identificação dos sujeitos, via de alimentação e pneumonias de repetição}

\begin{tabular}{|c|c|c|c|c|}
\hline Sujeitos & Idade & Gênero & Via de alimentação & $\begin{array}{c}\text { Pneumonias de } \\
\text { repetição }\end{array}$ \\
\hline S1 & $9 a 6 m$ & Feminino & Gastrostomia & Sim \\
\hline S2 & $2 a 6 m$ & Feminino & Via Oral (P., L.E., S.S.) & Não \\
\hline S3 & $1 a 2 m$ & Masculino & Via Oral (P.,L.E., L.) & Sim \\
\hline S4 & $3 a 8 m$ & Feminino & Via Oral (P., L.,S.S., S.) & Sim \\
\hline
\end{tabular}

Legenda 1 - P.=consistência pastosa; L=consistência líquida; L.E.=consistência líquida engrossada; S.=consistência sólida; S.S.=consistência semi-sólida.

O paciente com alimentação por gastrostomia foi encaminhado para verificar a possibilidade de reintrodução de alimentação por via oral e nos demais casos, as mães apresentavam queixas relacionadas com a alimentação, como: recusa alimentar, náuseas e vômitos após a alimentação, além de engasgos esporádicos para a consistência líquida.

Cabe ressaltar que S2, S3 e S4 apresentavam diagnóstico médico de doença do refluxo gastroesofágico (DRGE) e todos se encontravam sob tratamento medicamentoso. S1 não apresentava mais a doença no momento da avaliação fonoaudiológica, pois havia sido submetido à correção cirúrgica da DRGE, juntamente com a gastrostomia, indicada há cinco anos antes da avaliação fonoaudiológica.

Em análise retrospectiva dos protocolos fonoaudiológicos, foram coletados os dados da avaliação clínica do sistema sensório-motor orofacial, da avaliação clínica funcional e da avaliação videofluoroscópica da deglutição.

As avaliações clínicas do sistema sensóriomotor orofacial foram realizadas pela observação e/ ou toque na região orofacial, sendo analisados os seguintes aspectos: 
- Reflexos orais patológicos: presença, ausência ou exacerbação de reflexos orais patológicos de procura, mordida e vômito.

- Sensibilidade orofacial: hipersensibilidade, hipossensibilidade ou sensibilidade preservada.

- Tonicidade muscular orofacial geral: hipotonicidade, hipertonicidade ou normotonicidade.

- Sialorreia: presença ou ausência.

Para as avaliações clínicas funcionais da deglutição, foi solicitado que o responsável levasse o alimento de uso habitual da criança, assim como os utensílios para evitar recusa alimentar. Foram oferecidas as consistências pastosa (Danoninho® ou banana amassada) e líquida (água ou leite), nos volumes de 1 a $3 \mathrm{ml}$ em colher, copo ou seringa e $20 \mathrm{ml}$ para a deglutição contínua de consistência líquida oferecida em mamadeira.

Os aspectos analisados foram:

\section{Fase oral}

- Vedamento labial: ineficiente ou eficiente.

- Escape oral anterior: presença ou ausência.

\section{Fase faríngea}

- Deglutições múltiplas: presença (mais de três deglutições) ou ausência.

- Tosse: presença de tosse antes da deglutição; tosse durante a deglutição; tosse após a deglutição; ou ausência.

- Engasgo: presença o ausência.

- Alterações respiratórias: presença ou ausência.

- Ausculta cervical: ausculta alterada antes, durante ou após a deglutição; ou ausculta cervical adequada.

- Sinais sugestivos de penetração/aspiração laringotraqueal: presença (sinais de engasgo, tosse, lacrimejamento, alteração respiratória ou ausculta cervical alterada) ou ausência de sinais.

Para a avaliação videofluoroscópica da deglutição foram testadas as mesmas consistências e alimentos utilizados na avaliação clínica, adicionados ao contraste de bário (BarioGel $100 \% ®$ ), nos volumes de 1 a $3 \mathrm{ml}$ em colher, copo ou seringa e $20 \mathrm{ml}$ para a deglutição contínua de consistência líquida oferecida em mamadeira. Os exames foram realizados em vista látero-lateral, com a criança posicionada em bebê conforto. Os aspectos analisados foram:

\section{Fase oral}

- Formação/organização do bolo alimentar: inadequada ou adequada (formações dos tipos tipper ou dipper).
- Ejeção oral: ejeção deficiente, em sincronismo com a abertura da transição faringoesofágica (TFE); ejeção deficiente, sem sincronismo com a TFE; ejeção eficiente, sem sincronismo com a abertura da TFE; ejeção eficiente, em sincronismo com a abertura da TFE.

- Movimentação de língua: movimentos ânteroposteriores de língua ou póstero-anteriores.

- Controle oral: inadequado ou adequado.

- Resíduos em cavidade oral: presença ou ausência.

- Perda prematura do bolo para hipofaringe: presença ou ausência.

- Fracionamento do alimento: presença ou ausência.

\section{Fase faríngea}

- Eliciação faríngea: atrasada ou adequada.

- Refluxo nasal: presença ou ausência.

- Excursão hiolaríngea: inadequada ou adequada.

- Resíduos em trânsito faríngeo: presença ou ausência.

- Penetração laríngea: presença (antes, durante e/ou após a elevação da laringe) ou ausência.

- Aspiração laringotraqueal: presença (antes, durante e/ou após a elevação da laringe) ou ausência.

A avaliação videofluoroscópica foi complementar à avaliação clínica da deglutição. Sendo assim, foram selecionados apenas alguns itens presentes nos protocolos de avaliação clínica e videofluoroscópica, a fim de evitar a repetição de informações.

Com base nas avaliações clínica e videofluoroscópica da deglutição, foi realizada a classificação do grau de disfagia, seguindo os critérios de Silva ${ }^{11}$, que classificou as disfagias neurogênicas em leve, moderada e grave.

Os exames de videofluoroscopia da deglutição foram realizados no Setor de Radiologia do HCFMRP-USP, na presença de duas fonoaudiólogas e um técnico em radiologia, sendo utilizado o aparelho Pulsera (marca Phillips, modelo BV 300).

O presente estudo respeitou todas as normas estabelecidas pela resolução 196/96, acerca dos aspectos éticos em pesquisa com seres humanos, com aprovação do Comitê de Ética em Pesquisa do Hospital das Clínicas da Faculdade de Medicina de Ribeirão Preto - HCFMRP-USP, sob o processo de número 3970/2009.

Os resultados das avaliações de cada criança são apresentados de maneira descritiva, em forma de quadro. 


\section{RESULTADOS}

O Quadro 2 apresenta os resultados individuais das avaliações clínica do sistema sensório-motor orofacial, clínica funcional e videofluoroscópica da deglutição dos quatro sujeitos do estudo.

Com relação à avaliação da fase faríngea da deglutição, nenhum participante apresentou deglutições múltiplas e alterações respiratórias.
Em avaliação videofluoroscópica da deglutição, observou-se penetração e aspiração laringotraqueal para as consistências líquida e pastosa durante e após a deglutição em S1 e durante a deglutição em S3. Foi observada somente penetração laríngea durante a deglutição para a consistência líquida em S4.

A hipótese diagnóstica fonoaudiológica com base nos achados das avaliações de deglutição

\section{Quadro 2 - Resultados individuais das avaliações dos sujeitos}

\section{Sujeito 1: $\mathbf{S 1}$}

\section{Avaliação clínica do sistema sensório-motor} orofacial:

- Reflexo de mordida exacerbado; hipersensibilidade orofacial; normotonicidade orofacial.

\section{Avaliação clínica da deglutição:}

- Fase oral: vedamento labial ineficiente; escape oral anterior.

- Fase faríngea: presença de tosse (líquido); presença de engasgo (líquido); ausculta cervical alterada (líquido e pastoso).

\section{Avaliação videofluoroscópica da deglutição:}

- Fase oral: perda prematura do bolo; ejeção oral eficiente sem sincronismo com a abertura da TFE. - Fase faríngea: atraso na eliciação faríngea; excursão hiolaríngea reduzida; resíduo em trânsito faríngeo após deglutição; penetração laríngea (líquido e pastoso); aspiração laringotraqueal (líquido e pastoso).

Grau de disfagia: grave

\section{Sujeito 3: S3}

\section{Avaliação clínica do sistema sensório-motor orofacial:}

- Hipersensibilidade orofacial; hipertonicidade orofacial.

\section{Avaliação clínica da deglutição:}

- Fase oral: vedamento labial ineficiente; escape oral anterior.

- Fase faríngea: presença de tosse (líquido); ausculta cervical alterada (líquido).

\section{Avaliação videofluoroscópica da deglutição:}

- Fase oral: inadequada formação/organização do bolo; ejeção oral deficiente com sincronismo; movimento póstero-anterior de língua; inadequado controle oral; presença de resíduos em cavidade oral após deglutição; presença de fracionamento do alimento.

- Fase faríngea: presença de refluxo nasal; excursão hiolaríngea reduzida; resíduo em trânsito faríngeo após deglutição; penetração laríngea (líquido e pastoso); aspiração laringotraqueal (líquido e pastoso).

\section{Sujeito 2: S2}

\section{Avaliação clínica do sistema sensório-motor} orofacial:

- Hipersensibilidade orofacial; hipertonicidade orofacial.

\section{Avaliação clínica da deglutição:}

- Fase oral: vedamento labial ineficiente.

- Fase faríngea: ausculta cervical alterada (pastoso).

\section{Avaliação videofluoroscópica da deglutição:} - Fase oral: inadequada formação/organização do bolo; ejeção oral deficiente com sincronismo; movimento póstero-anterior de língua; inadequado controle oral. - Fase faríngea: excursão hiolaríngea reduzida; resíduo em trânsito faríngeo após deglutição.

Grau de disfagia: moderada

\section{Sujeito 4: S4}

\section{Avaliação clínica do sistema sensório-motor orofacial: \\ - Hipertonicidade orofacial.}

\section{Avaliação clínica da deglutição:}

- Fase oral: vedamento labial ineficiente; ausência de escape oral anterior.

- Fase faríngea: ausência de tosse e engasgo; ausculta cervical adequada.

\section{Avaliação videofluoroscópica da deglutição:}

- Fase oral: ejeção oral deficiente com sincronismo; presença de resíduos em cavidade oral após deglutição; presença de fracionamento do alimento.

- Fase faríngea: presença de refluxo nasal; excursão hiolaríngea reduzida; penetração laríngea (líquido).

Grau de disfagia: moderada

Grau de disfagia: grave 
para todas as crianças foi de disfagia orofaríngea neurogênica de graus moderado e grave.

\section{DISCUSSÃO}

No presente estudo, três sujeitos apresentaram hipersensibilidade orofacial e três apresentaram a musculatura orofacial hipertônica. Foi observado em um relato de caso ${ }^{9}$ de uma criança de 1 ano e 5 meses, com a síndrome Cornélia de Lange, aumento da sensibilidade nas regiões extra e intra-oral e hipertonia de lábios, língua e músculos bucinadores. Em outro trabalho ${ }^{7}$, verificou-se uma acentuada hipertonia dos órgãos fonoarticulatórios, comprometendo tanto a postura quanto a mobilidade, principalmente de lábios, língua e bochechas.

Apesar da maioria dos sujeitos deste estudo apresentar musculatura orofacial hipertônica, também foi encontrado um caso com presença de normotonia (S1), não descrita na literatura para a síndrome Cornélia de Lange. Essa condição pode ser decorrente do desuso da musculatura nas funções de deglutição e mastigação, visto que a criança encontrava-se em uso de via alternativa de alimentação por tempo prolongado.

As alterações encontradas, tanto na fase oral como na fase faríngea da deglutição em sujeitos do presente estudo, podem estar relacionadas às modificações na integridade do sistema nervoso central e nas estruturas envolvidas no processo de deglutição ${ }^{12}$. As alterações de tônus e sensibilidade orofacial, observadas nos resultados descritos e, inevitavelmente, na mobilidade das estruturas, contribuem para as dificuldades no processo de deglutição apresentadas por esses indivíduos.

A literatura é bastante escassa em casos de síndrome Cornélia de Lange relacionados com achados fonoaudiológicos, o que dificulta a comparação dos casos do presente estudo.

A maioria dos sujeitos com síndrome Cornélia de Lange do atual estudo apresentou ejeção oral do bolo alimentar deficiente. A ejeção oral nas disfagias orofaríngeas mostra-se, com frequência, deficiente devido à incapacidade de pressurização da cavidade oral. Nesse caso, a hipofaringe, quase sempre, mostra resíduo acentuado e não é incomum que penetração ou aspiração laringotraqueal estejam associadas a estas ejeções deficientes ${ }^{14}$

O sinal clínico de penetração/aspiração laringotraqueal na avaliação clínica de S1 e S3 foi confirmado pela avaliação objetiva. Outros autores ${ }^{14}$ também revelam essa relação entre as avaliações clínica e objetiva da deglutição. Sabe-se do importante papel da videofluoroscopia da deglutição, principalmente na identificação de aspiração silente ${ }^{15,16}$, que pode não ser evidenciada na avaliação clínica. Nesse sentido, sua indicação torna-se de grande valia na confirmação diagnóstica e caracterização das alterações na deglutição, bem como na definição de conduta terapêutica, em casos de síndrome Cornélia de Lange.

$\mathrm{Na}$ avaliação videofluoroscópica, foi comumente observada presença de refluxo nasal, excursão hiolaríngea reduzida, presença de resíduos em valécula e recessos piriformes e presença de penetração e aspiração laringotraqueal. Na literatura, há apenas relato de aspiração laringotraqueal para essa população ${ }^{3,6}$.

$O$ refluxo de alimento para a nasofaringe indica uma ineficiência do vedamento velofaríngeo e provoca escape de pressão durante a fase faríngea da deglutição, o que pode levar à aspiração laringotraqueal ${ }^{17}$. No presente estudo, o S3 apresentou tanto refluxo nasal como também aspiração laringotraqueal. Nesse caso, a presença de refluxo nasal, em conjunto com outras alterações apresentadas pelo sujeito, tanto em fase oral como em fase faríngea, pode ter contribuído para o episódio de aspiração. Diante da experiência clínica, a ocorrência de refluxo nasal observada em dois sujeitos do estudo, leva-nos a inferir que os pacientes com síndrome Cornélia de Lange podem apresentar inadequado vedamento velofaríngeo, com conseqüente refluxo nasal, determinado por diversos fatores, tanto anatômicos como funcionais.

A excursão hiolaríngea reduzida restringe a oclusão laríngea e reduz a proteção da via aérea, podendo ocasionar aspiração durante a deglutição ${ }^{18}$, como observado em S1 e S3. A limitada excursão laríngea observada em todos os sujeitos do estudo pode estar associada à alteração neuromuscular presente nos indivíduos com a síndrome Cornélia de Lange.

Além disso, sugere-se que a elevação laríngea reduzida associada à abertura limitada da transição faringoesofágica também ocasione a presença de alimentos retidos em valécula e nos recessos piriformes, em casos da síndrome. Isso pode ocorrer devido à hipertonia muscular e a redução na contração muscular da base da língua e parede faríngea ${ }^{17}$. Como verificado em avaliação, em dois sujeitos que apresentaram movimento pósteroanterior de língua na deglutição, foi evidenciado presença de resíduo em trânsito faríngeo após a deglutição. Esse movimento de língua pode reduzir a participação da base da língua na propulsão do bolo e favorecer a permanência de resíduos alimentares em valécula e recessos piriformes. Há risco de esses resíduos serem aspirados após a deglutição, principalmente para alimentos mais consistentes ${ }^{19}$, como observado na avaliação 
videofluoroscópica de $\mathrm{S} 1$, que aspirou resíduo alimentar de consistência pastosa.

Dois pacientes do presente estudo apresentaram aspiração laringotraqueal e três apresentaram penetração laríngea para a consistência líquida. Tal ocorrência é comum nas disfagias orofaríngeas, pois muitas vezes, o líquido é aspirado mais facilmente do que as consistências engrossadas, devido à tendência de fluir mais rapidamente com auxílio da gravidade e entrar em via aérea, como descrito em outros trabalhos ${ }^{15,17}$.

Observou-se em um caso da amostra (S3), a presença de aspiração laringotraqueal silente no exame de videofluoroscopia, isto é, o paciente aspirou as consistências pastosa e líquida oferecidas, sem a ocorrência de tosse ou engasgo, apesar de ter apresentado tosse durante a deglutição na avaliação clínica.

A aspiração silente refere-se à passagem de material deglutido dentro da via aérea sem ocorrência de sinais clínicos de limpeza, representada primeiramente por tosse. A falta de adequado reflexo de tosse é consequente de alteração no nível periférico, de inadequada coordenação dos músculos faríngeos a nível central, ou ainda, de reduzida sensibilidade faringolaríngea, o que pode explicar o elevado índice de aspiração silente em indivíduos com comprometimentos neurológicos ${ }^{20}$. Adicionalmente, a aspiração pode se tornar silenciosa devido ao tempo prolongado da passagem de saliva e alimento na laringe e na traquéia, provocando uma dessensibilização dos receptores sensitivos laríngeos ${ }^{21}$. Além de S3 apresentar comprometimento neurológico, é possível que o mesmo mantivesse episódios de aspiração por um longo período, contribuindo para a redução da sensibilidade. É comum, na prática clínica, os responsáveis de crianças com aspiração silente relatarem que as mesmas apresentavam episódios de engasgos e tosses, mas que naquele momento não apresentam mais. Isso quer dizer que a aspiração crônica diminui os sinais protetivos em decorrência da dessensibilização laríngea. No caso em questão, a responsável de S3 relatou, em anamnese, que a criança apresentava engasgos ocasionais apenas para a consistência líquida, sendo que foi evidenciada, em avaliação videofluoroscópica, a presença de aspiração também para a consistência pastosa.

A aspiração em crianças tem sido associada à significativa mortalidade e morbidade como pneumonias de repetição e desnutrição ${ }^{15,22}$. Foi verificado o histórico de pneumonias em três dos casos relatados, sendo confirmada aspiração laringotraqueal em dois destes (S1 e S3) por meio da videofluoroscopia. No caso de S1, não houve mais episódios de pneumonia após iniciar o uso exclusivo de sonda gástrica (gastrostomia) para a alimentação, conforme o relato da responsável.

Diante do exposto, cabe aos profissionais da equipe multidisciplinar permanecerem atentos quanto a possíveis alterações de deglutição relevantes em casos da síndrome, como a aspiração laringotraqueal, que pode ser silenciosa, e culminar em morbidades pulmonares e até em risco de morte. O conhecimento das alterações morfofuncionais, características da síndrome, é fundamental para se alcançar um diagnóstico precoce, bem como uma intervenção adequada. Ressalta-se a importância da atuação fonoaudiológica na tentativa de proporcionar melhor qualidade de vida a essa população.

Não foram encontrados trabalhos na literatura que, semelhantes a este, investigassem a biomecânica da deglutição por meio de avaliação clínica e videofluoroscópica em indivíduos com a síndrome Cornélia de Lange. São necessários mais estudos enfocando o aspecto da deglutição, uma vez que esses indivíduos apresentam alterações importantes neste mecanismo, como demonstrado pelo presente estudo.

\section{CONCLUSÃO}

Os achados encontrados apontaram alterações nos aspectos sensório-motores orofaciais e comprometimento na biomecânica da deglutição. As importantes alterações na deglutição evidenciadas nos casos descritos podem corresponder a manifestações típicas da síndrome Cornélia de Lange. 


\begin{abstract}
Purpose: to describe the findings for the oral and pharyngeal phases of swallowing, as well as the relevant orofacial sensorimotor aspects for children with Cornelia de Lange syndrome. Methods: This is a retrospective case report. We performed a descriptive analysis of four protocols for speech evaluation of swallowing, routinely used in the Speech Therapy Service and applied to children with Cornelia de Lange syndrome of both genders, with age going from 1:2 to 9:6 years, referred for clinical and videofluoroscopic evaluation of swallowing to a public university hospital. Aspects of the oral and pharyngeal of swallowing were analyzed using clinical functional and videofluoroscopic evaluation. Results: the changes in the orofacial sensorimotor system that were most detected are hypersensitivity and muscle hypertonia of the orofacial region. Among the changes observed in the oral phase of swallowing, was the presence of labial closure inefficient, previous oral escape of the food, inadequate bolus formation/organization and deficient oral ejection. In the pharyngeal phase there was a change in cervical auscultation, nasal reflux, reduced hyolaryngeal excursion, pharyngeal residue after deglutition, laryngeal penetration, and laryngotracheal aspiration. Oropharyngeal dysphagia was detected in all children. Conclusion: these findings indicate changes in the orofacial sensoriomotor aspects and impaired swallowing biomechanics. The important alterations in swallowing detected in these cases may correspond to typical manifestations of Cornelia de Lange syndrome.
\end{abstract}

KEYWORDS: De Lange Syndrome; Child; Deglutition; Deglutition Disorders

\section{REFERÊNCIAS}

1. Wiedemann HR, Kunze J, Dibbern H. Atlas de síndromes clínicas dismórficas. São Paulo: Manole; 1992. p.182-3.

2. Uzun $H$, Senses DA, Uluba M, Kocabay K. A newborn with Cornelia de Lange syndrome: a case report. Cases J. 2008; 1(1):329.

3. Ardón OG. Síndrome de Cornélia de Lange. Rev Hond Ped. 1986; 10(1):22-4.

4. Montes ML, Saldarriaga W, Isaza C. Descripción de un caso de síndrome de Cornelia de Lange. Aporte para un mejor diagnóstico pre-y post-natal. Rev Colomb Med. 2006; 37(4):323-7.

5. Palmero MI, Matute A, Rodríguez S, Marrugo M, Rojas R. Síndrome de Cornélia de Lange tipo III: a propósito de un caso. Revisión de literatura. Rev Obstet Ginecol Venez. 2007; 67(3):203-5.

6. Maia MMC. O papel dos profissionais da educação/reabilitação na promoção da resiliência na família de uma criança com Síndrome de Cornélia de Lange [monografia na Internet]. Porto: Escola Superior de Educação de Paula Frassinetti; 2008 [acesso em 2009 Jun 03]. Disponível em: http://purl.net/esepf/handle/10000/124.

7. Alves AG, Alves AA, Vieira MEM. G. Síndrome Cornélia de Lange: aspectos fonoaudiológicos. 15을 Simpósio Internacional de Iniciação Científica da Universidade de São Paulo; 2007. Ribeirão Preto: USP. CD ROM; 2007.
8. Sosa MP, Henao J. Sindrome de Cornelia de Lange. Rev Soc Bol Ped. 2005; 44(2):97-9.

9. Santos AM, Lopes AF, Curado ADF, Nakamura $\mathrm{NA}$, Eguti EY, Limongi SCO, et al. Relato de trabalho de orientação fonoaudiológica em uma criança portadora da Síndrome Cornélia de Lange. Pro-Fono. 1996; 8(1):3-6.

10. Fitzpatrick DR, Kline AD. Cornelia de Lange Syndrome. In: Cassidy SB, Allanson JE. Management of genetic syndromes. 2. ed. WileyLiss; 2005. p. 139-49.

11. Silva RG. Disfagia neurogênica em adultos pós-acidentes vascular encefálico: identificação e classificação. In: Disfagia: abordagem multidisciplinar. São Paulo: Frontis; 1998. p. 17-34.

12. Orenstein SR. Oral, pharyngeal, and esophageal motor disorders in infants and children. GI Motility Online [periódico on line]. 2006 [acesso em 06 Out 2009]; [about 62 p.]. Disponível em: URL: http:// www.nature.com/gimo/contents/pt1/full/gimo38. html.

13. Marrara JL, Duca AP, Dantas RO, Trawitzki LVV, Lima RAC, Pereira JC. Deglutição em crianças com alterações neurológicas: avaliação clínica e videofluoroscópica. Pró-Fono. 2008; 20(4):231-6.

14. Costa MMB. Avaliação da dinâmica da deglutição e da disfagia orofaringea. In: Castro S, Rocha MC. 10 Tópicos em gastroenterologia: deglutição e disfagia. Rio de Janeiro: MEDSI; 2000. p. 177-85. 
15. DeMatteo C, Matovich D, Hjartarson A. Comparison of clinical and videofluoroscopic evaluation of children with feeding and swallowing difficulties. Dev Med Child Neurol. 2005; 47(3):149-57.

16. Barbiera F, Condello S, De Palo A, Todaro D, Mandracchia C, De Cicco D. Role of videofluorography swallow study in management of dysphagia in neurologically compromised patients. Radiol Med. 2006; 111(6):818-27.

17. Langmore SE. Endoscopic evaluation of oral and pharyngeal phases of swallowing. GI Motility Online [periódico on line]. 2006 [acesso em 21 Set 2009]; [about 23 p.]. Disponível em: URL: http:// www.nature.com/gimo/contents/pt1/full/gimo28. html.

18. Logemann JA. Medical and rehabilitative therapy of oral, pharyngeal motor disorders. GI Motility Online [periódico on line]. 2006 [acesso em 22 Out 2009]; [about 11 p.]. Disponível em:
URL: http://www.nature.com/gimo/contents/pt1/full/ gimo50.html.

19. Massey BT, Shaker R. Oral, pharyngeal and upper esophageal sphincter motility disorders. GI Motility Online [periódico on line]. 2006 [acesso em 15 Ago 2009]; [about 18 p.]. Disponível em: URL: http://www.nature.com/gimo/contents/pt1/full/ gimo19.html.

20. Rugiu MG. Role of videofluoroscopy in evaluation of neurologic dysphagia. Acta Otorhinolaryngol Ital. 2007; 27(6):306-16.

21. Santini CS. Disfagia neurogênica. In: Furkim AM, Santini CS. Disfagias orofaríngeas. Carapicuíba: Pró-Fono; 2001. p. 19-34.

22. Cass $\mathrm{H}$, Wallis $\mathrm{C}$, Ryan $\mathrm{M}$, Reilly $\mathrm{S}$, McHugh $\mathrm{K}$. Assessing pulmonary consequences of dysphagia in children with neurological disabilities: when to intervene? Dev Med Child Neurol. 2005; 47(5):347-52.

DOI: 10.1590/S1516-18462010005000111

RECEBIDO EM: 09/12/2009

ACEITO EM: 23/07/2010

Endereço para correspondência:

Priscila Martins Foroni

Rua Rio Negro, 563

Ribeirão Preto - SP

CEP: 14060-170

E-mail: priscilaforoni@yahoo.com.br 